\title{
On the Existence of Thermodynamics for the Random Energy Model
}

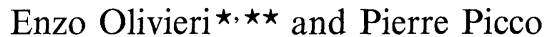

Centre de Physique Théorique, C.N.R.S. - Luminy - Case 907, F-13288 Marseille Cedex 9, France

\begin{abstract}
Derrida's random energy model is considered. Almost sure and $L^{P}$ convergence of the free energy at any inverse temperature $\beta$ are proven. Rigorous upper and lower bounds to the finite size corrections to the free energy are given.
\end{abstract}

\section{Introduction}

The Random Energy (R.E.) Model has been introduced by Derrida [I, II] as a simplified version of the mean field Sherrington-Kirkpatrick (S.K.) model [III] of a spin glass.

Both in the S.K. and in the R.E. models, the energies associated to each spin configuration in the volume $N$, are gaussian random variables with mean zero and covariance $N$.

In the S.K. model, we have an explicit microscopic hamiltonian, where the couplings are assumed to be independent gaussian normalized random variables so that the energies turn out to be dependent, whereas in the R.E. model, the microscopic hamiltonian is not specified and the energies are supposed to be independent random variables with the proper normalization. Thus the R.E. partition function has the following expression:

$$
Z_{N}=\sum_{i=1}^{2^{N}} \exp \beta \sqrt{N} X_{i} ; \quad X_{i} \in \mathscr{N}(0,1)
$$

In a recent paper, Eisele [IV] studied the R.E. model in a slightly more general situation by means of the theory of large deviations. He rigorously proved that the quenched free energy converges as $N \rightarrow \infty$, to a function $F(\beta)$ whose second derivative is discontinuous at $\beta=\beta_{c} \equiv \sqrt{2 \log 2}$ (third order phase transition). He was able to prove the almost sure convergence of the free energy only for $\beta \leqq \beta_{c}$, whereas he showed the stochastic convergence for any $\beta$. In the present paper we study the R.E. model by means of quite elementary techniques and establish the

* Université de Provence

$\star \star$ Permanent address: Dipartimento di Matematica Universita di Roma "La Sapienza" C.N.R. - G.N.F.M. 
almost sure convergence for any $\beta>0$ together with $L^{p}$ convergence for any $p \geqq 1$. We analyze the rate of convergence of the free energy and give explicit bounds to the finite size corrections that are of the expected form $\left(\sim \frac{\log N}{N}\right.$ for $\beta \geqq \beta_{c}$, $\approx \exp (-\operatorname{const} N)$ for $\left.\beta<\beta_{c}[\mathrm{II}]\right)$. We also show that the convergence is always geometric (see Definition 4.1 of [IV]) contrary to the conjecture formulated by Eisele (see remark after Theorem 5.4 of [IV]).

Let us now give a short description of the main idea of the proof. We divide the range of the random variables $X_{i}$, namely, the real line into intervals $\Delta_{K}$, and for any given sample $X_{1} \ldots X_{2^{n}}$, we treat separately the contribution to the partition function coming from the different intervals. It turns out that with high probability, the "occupation number" of an interval

$$
[X \sqrt{N} \sqrt{2 \log 2},(X+\Delta X) \sqrt{N} \sqrt{2 \log 2}[
$$

is of the order:

$$
2^{N} 2^{-N X^{2}} \text { for }|X|<1 \text {, and practically zero for }|X|>1,
$$

so that we are led to examine the function:

$$
G_{\beta}(X)=\left(1-X^{2}\right) \log 2+\beta \sqrt{2 \log 2} X, \quad|X|<1,
$$

which can be regarded as the contribution to the free energy per unit volume coming from the "level" $X$ in a typical sample. Now it is clear that the main contribution will come from the level $\bar{X}(\beta)$, where the function $G_{\beta}(X)$ gets its maximum value in the interval $[0,1]$ (the negative values of $X$ can be seen to be unimportant).

Therefore, it is reasonable to expect that the free energy converges as $N \rightarrow \infty$ to the function:

$$
F_{(\beta)}=G_{\beta}(\bar{X}(\beta)) .
$$

We rigorously prove this result in the case of the Derrida's (gaussian spin $\frac{1}{2}$ ) model for the sake of simplicity; but our method, that in fact goes back to the nonrigorous calculations made by Derrida [I, II], can be applied as well with minor changes to the class of models considered in [IV].

In Sect. II, we state the results in a precise form (Theorem I and Propositions 2-5).

The proof of Theorem I is very simple and it is contained in Sect. III.

The proof of Propositions 2-5, which is little more lengthy, is given in Sect. IV.

\section{Definitions and Results}

Let $(\Omega, \Sigma, \mathbb{P})$ be a probability space. We assume that for any $N \in \mathbb{N}$ there exists a family $\left\{X_{i}\right\}, i=1 \ldots 2^{N}$ of independent normalized gaussian random variables that are defined on $(\Omega, \Sigma, \mathbb{P})$.

Let $\beta$ be a positive real number; we define the random variables:

$$
Z_{N}(\beta)=\sum_{i=1}^{2^{N}} \exp \left(\beta \sqrt{N} X_{i}\right)
$$


and the random variables:

$$
F_{N}(\beta)=\frac{1}{N} \log Z_{N}(\beta)
$$

(finite volume free energy).

Define also: $\beta_{c}=\sqrt{2 \log 2}$ and

$$
F(\beta)=\left\{\begin{array}{lll}
\beta^{2} / 2+\beta_{c}^{2} / 2 & \text { if } & 0 \leqq \beta \leqq \beta_{c} \\
\beta \beta_{c} & \text { if } & \beta \geqq \beta_{c} .
\end{array}\right.
$$

Our main result is:

Theorem 1. $\forall \beta>0$,

$$
\lim _{N \rightarrow \infty} F_{N}(\beta)=F(\beta) \quad \text { almost surely }
$$

and in $L^{p}(\Omega, \Sigma, \mathbb{P})$ for any $1 \leqq p<\infty$.

The following propositions summarize our results about finite size corrections:

Proposition 2. Let $\beta \geqq \beta_{c}$, then for almost all $\omega \in \Omega$ there exists $N_{-}(\omega)$ such that for all $N \geqq N_{-}(\omega)$

$$
F_{N}(\beta) \geqq F(\beta)-\frac{1}{2 \beta_{c}^{2}} \frac{\log N}{N}+o\left(\frac{\log N}{N}\right) .
$$

Moreover,

$$
\mathbb{E}\left(F_{N}(\beta)\right) \geqq F(\beta)-\frac{1}{2 \beta_{c}^{2}} \frac{\log N}{N}+o\left(\frac{\log N}{N}\right),
$$

for all sufficiently large $N$.

Proposition 3. Let $\beta \geqq \beta_{o}$, then for all positive $\delta$ and for almost all $\omega \in \Omega$ there exists $N_{+}(\omega, \delta)$ such that for any $N \geqq N_{+}(\omega, \delta)$,

$$
F_{N}(\beta) \leqq F(\beta)+\left(\frac{1}{2 \beta_{c}^{2}}+\delta\right) \frac{\log N}{N}+o\left(\frac{\log N}{N}\right) .
$$

Moreover,

$$
\mathbb{E}\left(F_{N}(\beta)\right) \leqq F(\beta)+\left(\frac{1}{2 \beta_{c}^{2}}+\delta\right) \frac{\log N}{N}+o\left(\frac{\log N}{N}\right)
$$

for all sufficiently large $N$.

Proposition 4. Let $\beta \geqq \beta_{c}$, then for any $\varepsilon>0$,

$$
\lim _{N \rightarrow \infty} \mathbb{P}\left(F_{N}(\beta)>F(\beta)-\frac{1}{2 \beta_{c}^{2}}(1+\varepsilon) \frac{\log N}{N}\right)=0 .
$$

Proposition 5. Let $\beta<\beta_{c}$, then there exists a positive number $\lambda(\beta)$ and for almost all $\omega \in \Omega$, there exists an $\bar{N}(\omega)$ such that: for any $N \geqq \bar{N}(\omega)$

$$
F(\beta)-\exp -\lambda(\beta) N \leqq F_{N}(\beta) \leqq F(\beta)+\exp -\lambda(\beta) N .
$$


Moreover,

$$
F(\beta)-\exp -\lambda(\beta) N \leqq \mathbb{E}\left(F_{N}(\beta)\right) \leqq F(\beta)+\exp -\lambda(\beta) N
$$

for all sufficiently large $N$.

We notice that the lower bound on the quenched free energy given by Eq. II.5 coincides with the first two terms of the asymptotic expansion given by Derrida [II]; in other words, the constant in front of $\frac{\log N}{N}$ that we found is in some sense optimal. Moreover, the result given by Proposition 4 is indeed an upper bound in probability to the free energy which contains exactly the same (negative) finite size correction. This is a further indication that in fact, the right finite size correction is given at the lowest order by

$$
-\frac{1}{2 \beta_{c}^{2}} \frac{\log N}{N}
$$

Unfortunately, we are not able to prove the almost sure analogous statement, but in any case, the result given by Proposition 3 enables us to say that the order of the finite size correction is almost surely at most $\frac{\log N}{N}$ so that, for example, we can exclude $\frac{1}{\sqrt{N}}$ corrections in a rigorous way.

Finally, we remark that, for $\beta<\beta_{c}$ exponentially small, corrections were already obtained by Derrida for the quenched free energy [II].

\section{Proof of Theorem 1}

The proof of Theorem 1 is based on the following decomposition of the real line: Let $M \in \mathbb{N} \backslash\{0\}$. We set:

$$
\mathbb{R}=\bigcup_{K=-1}^{M+1} \Delta_{K}
$$

where

$$
\begin{gathered}
\left.\left.\Delta_{-1}=\right]-\infty 0\right], \\
\left.\left.\Delta_{K}=\right] K \frac{\sqrt{N} \beta_{c}}{M},(K+1) \frac{\sqrt{N}}{M} \beta_{c}\right] \quad \text { if } \quad 0 \leqq K \leqq M, \\
\left.\Delta_{M+1}=\right]\left(1+\frac{1}{M}\right) \sqrt{N} \beta_{c},+\infty[.
\end{gathered}
$$

We call $\Delta_{K}$ the energy levels and study the occupation times of these energy levels, namely: The integer valued random variables:

$$
N_{K}=\sum_{i=1}^{2^{N}} \mathbb{1}_{\Delta_{K}}\left(X_{i}\right)=\#\left(i / X_{i} \in \Delta_{K}\right) .
$$

We first prove the following lemma. 
Lemma III.1. Let $\alpha_{N}=\exp \frac{N}{M}$, then

$$
\sum_{N=1}^{\infty} M \operatorname{Sup}_{0 \leqq K \leqq M} \mathbb{P}\left(N_{K}>\alpha_{N} 2^{N} \mathbb{E}\left(\mathbb{1}_{\Delta_{K}}(x)\right)\right)<\infty .
$$

Proof. By Markov's inequality (V) we get:

$$
\begin{aligned}
\operatorname{Prob}\left(N_{K}>\alpha_{N} 2^{N} \mathbb{E}\left(\mathbb{1}_{\Delta_{K}}\right)\right) & =\operatorname{Prob}\left(N_{K}>\alpha_{N} \mathbb{E}\left(N_{K}\right)\right) \\
& \leqq \exp -\frac{N}{M}
\end{aligned}
$$

Hence the lemma follows.

Now, if we define:

$$
\Omega_{N}=\bigcap_{K=0}^{M}\left\{\omega \in \Omega / N_{K}(\omega) \leqq \alpha_{N} 2^{N} \mathbb{E}\left(\mathbb{1}_{\Delta_{K}}\right)\right\},
$$

the Borel Cantelli Lemma (V) and Lemma III.1 imply that:

$$
\operatorname{Prob}\left(\overline{\lim } \Omega_{N}^{c}\right)=0 \text {. }
$$

Now, we want to prove that for any given $\varepsilon>0$,

$$
\varlimsup \frac{1}{N} \log Z_{N} \leqq(1+\varepsilon) F(\beta) \quad \text { almost surely } .
$$

In order to do that, we decompose the partition function in the following way:

$$
\begin{aligned}
\sum_{i=1}^{2^{N}} \exp \sqrt{N} \beta X_{i}= & \sum_{i=1}^{2^{N}}\left(\exp \sqrt{N} \beta X_{i}\right)\left(\mathbb{1}_{\Delta_{-1}}\left(X_{i}\right)+\mathbb{1}_{\Delta_{M+1}}\left(X_{i}\right)\right) \\
& +\sum_{K=0}^{M} \sum_{i=1}^{2^{N}} \mathbb{1}_{\Delta_{K}}\left(X_{i}\right) \exp \sqrt{N} \beta X_{i} .
\end{aligned}
$$

We first consider the last sum in the right-hand side of (III.7). As we have seen before, we have that for almost all $\omega$ there exists an $N_{1}(\omega)$ such that for any $N \geqq N_{1}(\omega)$ and for any $K=0,1, \ldots, M$ :

$$
\sum_{K=0}^{M} \sum_{i=1}^{2^{N}} \mathbb{1}_{\Delta_{K}}\left(X_{i}\right) \exp \sqrt{N} \beta X_{i} \leqq \sum_{K=0}^{M} \alpha_{N} 2^{N} \mathbb{E}\left(\mathbb{1}_{\Delta_{K}}\right) \exp \sqrt{N} \beta\left[(K+1) \frac{\sqrt{N} \beta_{c}}{M}\right]
$$

Since

$$
\mathbb{E}\left(\mathbb{1}_{\Delta_{K}}\right) \leqq \exp -\frac{K^{2} N \beta_{c}^{2}}{2 M^{2}}
$$

the right-hand side of (III.8) does not exceed

$$
\sum_{K=0}^{M} \alpha_{N} \exp N\left[\beta \beta_{c} \frac{(K+1)}{M}+\frac{\beta_{c}^{2}}{2}\left(1-\frac{K^{2}}{M^{2}}\right)\right]
$$

Now recalling that

$$
G_{\beta}(x)=\beta \beta_{c} x+\frac{\beta_{c}^{2}}{2}\left(1-x^{2}\right),
$$


we get

$$
\operatorname{Max}_{x \in[0,1]} G_{\beta}(x)=F(\beta) ;
$$

then, if $N \geqq N_{1}(\omega)$, (III.9) does not exceed

$$
\alpha_{N} M \exp \left(N F(\beta)+\frac{N}{M} \beta \cdot \beta_{c}\right)
$$

On the other hand,

$$
\sum_{i=1}^{2^{N}} \mathbb{1}_{\Delta-1}\left(X_{i}\right) \exp \sqrt{N} \beta X_{i} \leqq 2^{N}=\exp N \frac{\beta_{c}^{2}}{2} \leqq \exp N F(\beta)
$$

[since if $\beta \leqq \beta_{c} F(\beta)=\beta_{c}^{2} / 2+\beta^{2} / 2>\beta_{c}^{2} / 2$ if $\beta>\beta_{c} F(\beta)=\beta \cdot \beta_{c}>\beta_{c}^{2} / 2$ ].

Finally, we consider:

$$
\sum_{i=1}^{2^{N}} \mathbb{1}_{\Delta_{M+1}}\left(X_{i}\right) \exp \sqrt{N} \beta X_{i}
$$

By using Markov inequality (V) we get:

$$
\begin{aligned}
\operatorname{Prob}\left(\sum_{i=1}^{2^{N}} \mathbb{1}_{\Delta_{M+1}} \geqq \frac{1}{2}\right) & \leqq 2^{N+1} \exp -N \frac{\beta_{c}^{2}}{2}\left(1+\frac{1}{M}\right)^{2} \\
& \leqq 2 \exp -\frac{N}{M} \beta_{c}^{2} .
\end{aligned}
$$

Therefore, the Borel Cantelli lemma implies that for almost all $\omega$ there exists an $N_{2}(\omega)$ such that for any $N \geqq N_{2}(\omega): 0 \leqq \sum_{i=1}^{2^{N}} \mathbb{1}_{\Delta_{M+1}}\left(X_{i}\right) \leqq \frac{1}{2}$.

Since $\sum_{i=1}^{2^{N}} \mathbb{1}_{\Delta_{M+1}}\left(X_{i}\right)$ can only take integer values, it follows that it is necessarily equal to zero for $N \geqq N_{2}(\omega)$. This fact together with the inequality:

$$
0 \leqq \sum_{i=1}^{2^{N}} \mathbb{1}_{\Delta_{M+1}}\left(X_{i}\right) \exp \sqrt{N} \beta X_{i} \leqq\left[\exp \left(\sqrt{N} \beta \underset{1 \leqq i \leqq 2^{N}}{\operatorname{Max}} X_{i}\right)\right] \sum_{i=1}^{2^{N}} \mathbb{1}_{\Delta_{M+1}}\left(X_{i}\right)
$$

implies that for almost all $\omega$ if $N \geqq N_{2}(\omega)$,

$$
\sum_{i=1}^{2^{N}} \mathbb{1}_{\Delta_{M+1}}\left(X_{i}\right) \exp \sqrt{N} \beta X_{i}=0 .
$$

By Eqs. (III.11), (III.12), and (III.16), we eventually get: for almost all $\omega$ there exist an $N(\omega)=\operatorname{Max}\left(N_{1}(\omega), N_{2}(\omega), N_{0}(\varepsilon)=\frac{2 \log 2 M}{\varepsilon}\right)$, such that for all $N \geqq N(\omega)$ if $M>\frac{2\left(2+\beta \cdot \beta_{c}\right)}{\varepsilon}$,

$$
\frac{1}{N} \log Z_{N} \leqq(1+\varepsilon) F(\beta)
$$


Now we prove that for any $\varepsilon>0$

$$
\underline{\lim } \frac{1}{N} \log Z_{N} \geqq(1-\varepsilon) F(\beta)
$$

almost surely.

The idea of the proof is very simple: we bound from below the partition function by only keeping the contribution coming from the level near to the point $\bar{x}=\bar{x}(\beta)$, where the function $G_{\beta}(x)$ [see (III.10)] gets its maximum value in the interval $[0,1]$.

Notice that

$$
\bar{x}(\beta)= \begin{cases}\beta / \beta_{c} & \text { if } \quad 0 \leqq \beta \leqq \beta_{c} \\ 1 & \text { if } \quad \beta \geqq \beta_{c}\end{cases}
$$

We can write,

$$
\text { for all } \omega \sum_{i=1}^{2^{N}} \exp \sqrt{N} \beta X_{i} \geqq \sum_{i=1}^{2^{N}} \mathbb{1}_{\Delta_{\overline{\mathrm{K}}}}\left(X_{i}\right) \exp N \beta \beta_{c}\left(\bar{x}-\frac{1}{M}\right) \text {, }
$$

where $\bar{K}=[M \bar{x}]-1$ if $M \geqq \beta_{c} / \beta$.

Using Tchebychef's inequality we get

$$
\begin{aligned}
& \operatorname{Prob}\left(\sum_{i=1}^{2^{N}} \mathbb{1}_{\Delta_{\bar{K}}}\left(X_{i}\right) \leqq \frac{1}{2} 2^{N} \mathbb{E}\left(\mathbb{1}_{\Delta_{\bar{K}}}\right)\right) \\
& \leqq \operatorname{Prob}\left(\left|\sum_{i=1}^{2^{N}} \mathbb{1}_{\Delta_{\bar{K}}}\left(X_{i}\right)-2^{N} \mathbb{E}\left(\mathbb{1}_{\Delta_{\bar{K}}}\right)\right| \geqq \frac{1}{2} 2^{N} \mathbb{E}\left(\mathbb{1}_{\Delta_{\bar{K}}}\right)\right) \\
& \leqq\left(2^{N-1} \mathbb{E}\left(\mathbb{1}_{\Delta_{\bar{K}}}\right)\right)^{-2} \mathbb{E}\left(\left\{\sum_{i=1}^{2^{N}}\left(\mathbb{1}_{\Delta_{\bar{K}}}\left(X_{i}\right)-\mathbb{E}\left(\mathbb{1}_{\Delta_{\bar{K}}}\right)\right\}^{2}\right)\right. \\
& \leqq\left(2^{N-2} \mathbb{E}\left(\mathbb{1}_{\Delta_{\bar{K}}}\right)\right)^{-1} .
\end{aligned}
$$

Now it is not difficult to see that

$$
\mathbb{E}\left(\mathbb{1}_{\Delta_{\bar{K}}}\right) \geqq \mathbb{E}\left(\mathbb{1}_{\Delta_{M-1}}\right) .
$$

On the other hand, the right-hand side of (III.22) is bigger than

$$
\int_{\left(1-\frac{1}{M}\right) \sqrt{N} \beta_{c}}^{\left(1-\frac{1}{2 M}\right) \sqrt{N} \beta_{c}} \exp -\frac{x^{2}}{2} \frac{d x}{\sqrt{2 \pi}} \geqq \frac{\sqrt{N}}{2 M} \frac{\beta_{c}}{\sqrt{2 \pi}} \exp -\frac{N \beta_{c}^{2}}{2}\left(1-\frac{1}{2 M}\right)^{2},
$$

therefore, since

$$
\sum_{N=1}^{\infty} \frac{M}{\sqrt{N}} 2^{-N} e^{\frac{1}{2}\left(1-\frac{1}{2 M}\right)^{2} N \beta_{c}^{2}} \leqq \sum_{N=1}^{\infty} \frac{M}{\sqrt{N}} \exp -\frac{\beta_{c}^{2}}{4} \frac{N}{M}<\infty,
$$

the Borel Cantelli lemma implies that for almost all $\omega$ there exists an $N_{3}(\omega)$ such that for all $N \geqq N_{3}(\omega)$ :

$$
\sum_{i=1}^{2^{N}} \mathbb{1}_{\Delta_{\bar{K}}}\left(X_{i}\right) \geqq 2^{N-1} \mathbb{E}\left(\mathbb{1}_{\Delta_{\bar{K}}}\right) \geqq 2^{N-1} \frac{\sqrt{N}}{2 M} \frac{\beta_{c}}{\sqrt{2 \pi}} \exp -\frac{N \beta_{c}^{2}}{2} \bar{x}^{2} .
$$


Therefore, for almost all $\omega$ if $N \geqq N_{3}(\omega)$,

$$
\sum_{i=1}^{2^{N}} e^{\sqrt{N \beta X_{i}}} \geqq\left(\exp N\left\{F(\beta)-\beta \cdot \beta_{c} \frac{1}{M}\right\}\right) \cdot \frac{\sqrt{N}}{4 M \sqrt{2 \pi}},
$$

and this concludes the proof of the first part of Theorem 1.

In order to prove the second part of Theorem 1, we use the mean convergence criterion (V): If the random variables $\left\{\left|Y_{n}\right|^{p}, n \geqq 1\right\}$ are uniformly integrable (u.i.), that is

$$
\lim _{\alpha \rightarrow \infty} \operatorname{Sup}_{n \geqq n 0} \int_{\left|Y_{n}\right|^{p} \geqq \alpha}\left|Y_{n}\right|^{p} d \mathbb{P}=0,
$$

and $Y_{n} \rightarrow Y$ in probability, then $Y_{n} \rightarrow Y$ in $L^{P}$.

We start from the inequalities:

$$
\beta \frac{1}{\sqrt{N}} \operatorname{Max}_{1 \leqq i \leqq 2^{N}} X_{i} \leqq \frac{1}{N} \log Z_{N} \leqq \beta \frac{1}{\sqrt{N}} \operatorname{Max}_{1 \leqq i \leqq 2^{N}} X_{i}+\log 2
$$

From (III.28) we have if $\alpha \geqq 1$,

$$
\begin{aligned}
& \int_{\left|F_{N}(\beta)\right|^{p}>\alpha}\left|F_{N}(\beta)\right|^{p} d \mathbb{P} \leqq \int_{F_{N}(\beta)>\alpha}\left(F_{N}(\beta)\right)^{p} d \mathbb{P}+\int_{F_{N}(\beta)<-\alpha}\left(-F_{N}(\beta)\right)^{p} d \mathbb{P}
\end{aligned}
$$

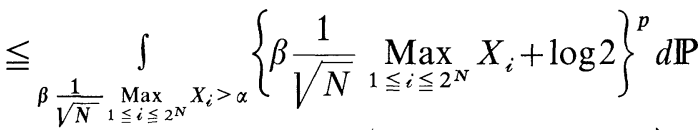

$$
\begin{aligned}
& +\int_{\log 2+\beta \frac{1}{\sqrt{N}} \operatorname{Max}_{1 \leqq i \leqq 2^{N}} X_{i}<-\alpha}\left(-\beta \frac{1}{\sqrt{N}} \operatorname{Max}_{1 \leqq i \leqq 2^{N}} X_{i}\right)^{p} d \mathbb{P} .
\end{aligned}
$$

In order to estimate (III.30), we use the following asymptotic estimate for

$$
\begin{aligned}
\operatorname{Prob}\left(\frac{1}{\sqrt{N}} \operatorname{Max}_{1 \leqq i \leqq 2^{N}} X_{i}>\frac{\alpha}{\beta} K\right) & =1-\left[1-\operatorname{Prob}\left(X>\frac{\alpha K}{\beta} \sqrt{N}\right)\right]^{2^{N}} \\
& \leqq 2^{N} \exp -\frac{\alpha^{2} K^{2} N}{2 \beta^{2}}
\end{aligned}
$$

which implies that (III.30) does not exceed

$$
\begin{gathered}
\sum_{K=1}^{\infty}\left(\frac{\alpha}{\beta}(K+1)+\log 2\right)^{p} 2^{N} \exp -\frac{\alpha^{2} K^{2} N}{2 \beta^{2}} \\
\leqq\left(\frac{\alpha}{\beta}+\log 2\right)^{p} \sum_{K=1}^{\infty}(K+1)^{p} \exp -\frac{\alpha^{2} K^{2}}{4 / 3^{2}} .
\end{gathered}
$$

(III.34) goes to zero as $\alpha$ goes to infinity.

In order to estimate (III.31), here we use:

$$
\begin{aligned}
\operatorname{Prob}\left(\beta \frac{1}{\sqrt{N}} \operatorname{Max}_{1 \leqq i \leqq 2^{N}} X_{i}<-K(\alpha+\log 2)\right) & =\left[\operatorname{Prob}\left(X \leqq-K(\alpha+\log 2) \frac{\sqrt{N}}{\beta}\right)\right]^{2^{N}} \\
& \leqq \exp -(\alpha+\log 2)^{2} \frac{N}{2 \beta^{2}} 2^{N} K^{2},
\end{aligned}
$$


which implies that (III.31) does not exceed

$$
\begin{gathered}
\sum_{K=1}^{\infty}((\alpha+\log 2)(K+1))^{p} \exp -(\alpha+\log 2)^{2} \frac{N 2^{N} K^{2}}{2 \beta^{2}} \\
\leqq(\alpha+\log 2)^{p} \sum_{K=1}^{\infty}(K+1)^{p} \exp -(\alpha+\log 2)^{2} \frac{K^{2}}{2 \beta^{2}},
\end{gathered}
$$

which goes to zero as $\alpha$ goes to infinity.

\section{Proof of Propositions 2-5}

\section{Proof of Proposition 2}

We start from the following inequality:

$$
Z_{N} \geqq \sum_{i=1}^{2^{N}} \mathbb{1}_{\Delta}\left(X_{i}\right) \exp \sqrt{N} \beta X_{i},
$$

where $\Delta$ is the interval $\left[\sqrt{N} \beta_{c}\left(1-a \frac{\log N}{N}\right), \sqrt{N} \beta_{c}\right], a$ is a positive number depending on $N$ that will be chosen later. We get

$$
Z_{N} \geqq\left\{\exp N\left(F(\beta)-\beta \cdot \beta_{c} a \frac{\log N}{N}\right)\right\} \sum_{i=1}^{2^{N}} \mathbb{1}_{\Delta}\left(X_{i}\right) .
$$

Now it is clear that the first part of Proposition 2 will be an immediate consequence of the Borel-Cantelli lemma and of the following:

Lemma 6. Define

$$
\begin{aligned}
& a=\frac{1}{2 \beta_{c}^{2}}+\frac{1}{\log \log N}, \\
& b=\frac{2 \beta_{c}^{2}}{\log N}-\frac{\beta_{c}^{2}}{\log \log N} .
\end{aligned}
$$

Then

$$
\sum_{N=1}^{\infty} \mathbb{P}\left(\sum_{i=1}^{2^{N}} \mathbb{1}_{\Delta}\left(X_{i}\right) \leqq e^{-b \log N}\right)<\infty .
$$

Proof. If the following condition on $a$ and $b$ is satisfied:

$$
\exp -b \log N \leqq \frac{1}{2} 2^{N} \mathbb{E}\left(\mathbb{1}_{\Delta}\right)
$$

then

$$
\begin{aligned}
& \operatorname{Prob}\left(\sum_{i=1}^{2^{N}} \mathbb{1}_{\Delta}\left(X_{i}\right) \leqq \exp -b \log N\right) \\
& \quad \leqq \operatorname{Prob}\left(\left|\sum_{i=1}^{2^{N}} \mathbb{1}_{\Delta}\left(X_{i}\right)-\mathbb{E}\left(\mathbb{1}_{\Delta}\left(X_{i}\right)\right)\right| \geqq 2^{N-1} \mathbb{E}\left(\mathbb{1}_{\Delta}\right)\right),
\end{aligned}
$$

The right-hand side of Eq. (IV.6) can be estimated by means of the Bernstein's inequality $[\mathrm{VI}]$ : 
Suppose $Y_{i}$ are independent random variables with mean zero, $\left|Y_{i}\right| \leqq 1$. If $D_{n}=\sum_{i=1}^{n} \mathbb{E}\left(Y_{i}^{2}\right)$ and if $0<t<\sqrt{D_{n}}$, then

$$
\operatorname{Prob}\left(\left|\sum_{i=1}^{n} Y_{i}\right| \geqq t \sqrt{D_{n}}\right) \leqq 2 \exp -\frac{t^{2}}{2} .
$$

We choose $t=\sqrt{2^{N-1} \mathbb{E}\left(\mathbb{1}_{\Delta}\right)}$. Since $D_{N}=2^{N}\left(\mathbb{E}\left(\mathbb{1}_{\Delta}\right)-\left[\mathbb{E}\left(\mathbb{1}_{\Delta}\right)\right]^{2}\right), \quad \mathbb{E}\left(\mathbb{1}_{\Delta}\right) \leqq \frac{1}{2}$, and so

$$
2^{N-1} \mathbb{E}\left(\mathbb{1}_{\Delta}\right) \leqq D_{N} \leqq 2^{N} \mathbb{E}\left(\mathbb{1}_{\Delta}\right)
$$

we get

$$
\begin{aligned}
& \operatorname{Prob}\left(\left|\sum_{i=1}^{2^{N}} \mathbb{1}_{\Delta}\left(X_{i}\right)-\mathbb{E}\left(\mathbb{1}_{\Delta}\left(X_{i}\right)\right)\right| \geqq 2^{N-1} \mathbb{E}\left(\mathbb{1}_{\Delta}\right)\right) \\
& \quad \leqq 2 \exp -\frac{1}{4} 2^{N} \mathbb{E}\left(\mathbb{1}_{\Delta}\right) .
\end{aligned}
$$

Now, in order to prove the lemma, it is sufficient to find a lower bound $\lambda_{N}$ to $2^{N} \mathbb{E}\left(\mathbb{1}_{\Delta}\right)$ such that:

i)

$$
\lambda_{N} \geqq 2 \exp -b \log N
$$

[so that condition (IV.5) is satisfied], and

ii)

$$
\sum_{N=0}^{\infty} \exp -\frac{\lambda_{N}}{4}<\infty
$$

Now it is easily seen that if $0 \leqq \theta_{N}=a$, then

$$
2^{N} \mathbb{E}\left(\mathbb{1}_{\Delta}\right) \geqq \lambda_{N} \equiv \beta_{c} \theta_{N} \log N \cdot \exp \left[N \frac{\beta_{c}^{2}}{2}\left(2\left(a-\theta_{N}\right)-\frac{1}{\beta_{c}^{2}}\right) \frac{\log N}{N}\right] .
$$

In fact, by definition:

$$
\mathbb{E}\left(\mathbb{1}_{\Delta}\right) \geqq \int_{\sqrt{N} \beta_{c}\left(1-a \frac{\log N}{N}\right)}^{\sqrt{N} \beta_{c}\left(1-\left(a-\theta_{N}\right) \frac{\log N}{N}\right)} \exp -\frac{x^{2}}{2} \frac{d x}{\sqrt{2 \pi}}
$$

from which estimate (IV.11) immediately follows.

Now if we choose $\theta_{N}=\frac{1}{\log N}$ it is easy to check that conditions i) and ii) in
10) are satisfied. (IV.10) are satisfied.

In order to prove the second part of Proposition 2, namely, the inequality (II.5) we start from:

$$
\mathbb{E}\left(F_{N}(\beta)\right)=\mathbb{E}\left(\mathbb{1}_{\Omega^{-}} F_{N}(\beta)\right)+\mathbb{E}\left(\mathbb{1}_{\Omega^{+}} F_{N}(\beta)\right),
$$

where

$$
\Omega^{-}=\left\{\log Z_{N}<0\right\}, \quad \Omega^{+}=\left\{\log Z_{N} \geqq 0\right\},
$$

using

$$
F_{N}(\beta) \geqq \frac{\beta}{\sqrt{N}} \operatorname{Max}_{1 \leqq i \leqq 2^{N}} X_{i} \equiv \beta \cdot \beta_{c}\left(\frac{\underset{1 \leqq i \leqq 2^{N}}{\operatorname{Max} X_{i}}}{\sqrt{2 \log 2^{N}}}\right),
$$


if

$$
B=\left\{\operatorname{Max}_{1 \leqq i \leqq 2^{N}} X_{i} \leqq 0\right\}
$$

By Schwarz' inequality we get:

since

$$
\begin{aligned}
\mathbb{E}\left(\left|\mathbb{1}_{\Omega^{-}} F_{N}(\beta)\right|\right) & \leqq \beta \cdot \beta_{c} \mathbb{E}\left(\left|\mathbb{1}_{B} \frac{\operatorname{Max}_{\underline{1} 2^{N}} X_{i}}{\sqrt{2 \log 2^{N}}}\right|\right) \\
& \leqq \beta \cdot \beta_{c}\left[\mathbb{E}\left(\mathbb{1}_{B}\right)\right]^{1 / 2}\left\{\mathbb{E}\left[\left(\frac{1 \leqq i \leqq 2^{N}}{\sqrt{2 \log 2^{N}}}\right)^{2}\right]\right\}^{1 / 2},
\end{aligned}
$$

$$
\mathbb{E}\left(\mathbb{1}_{B}\right) \leqq\left(\frac{1}{2}\right)^{2^{N}} \text { and } \operatorname{Max}_{1 \leqq i \leqq 2^{N}} X_{i} / \sqrt{2 \log 2^{N}}
$$

is in $L^{2}(\Omega, \Sigma, \mathbb{P})$ [as it can be seen by using (III.33) and (III.35)]. Then

$$
\mathbb{E}\left(\mathbb{1}_{\Omega^{-}} F_{N}(\beta)\right) \geqq- \text { const } \exp -2^{N} \log 2 .
$$

By the previous estimates [Eqs. (IV.9), (IV.11)] we know that:

$$
\begin{gathered}
\text { if } \hat{\Omega}_{N}=\left\{\sum_{i=1}^{2^{N}} \mathbb{1}_{\Delta}\left(X_{i}\right) \geqq \frac{1}{2} 2^{N} \mathbb{E}\left(\mathbb{1}_{\Delta}\right)\right\} \\
\mathbb{E}\left(\mathbb{1}_{\hat{\Omega}_{N}}\right) \geqq 1-\exp -c N^{\frac{\alpha}{\log \log N}} \text { for some } c \text { and } \alpha .
\end{gathered}
$$

Therefore,

$$
\begin{aligned}
\mathbb{E}\left(\mathbb{1}_{\Omega_{+}} F_{N}(\beta)\right) \geqq & \mathbb{E}\left(\mathbb{1}_{\Omega_{+}} \mathbb{1}_{\hat{\Omega}_{N}} F_{N}(\beta)\right) \\
\geqq & {\left[F(\beta)-\frac{1}{2 \beta_{c}^{2}} \frac{\log N}{N}+o\left(\frac{\log N}{N}\right)\right] } \\
& \cdot\left[1-\text { const } \exp -2^{N} \log 2-\exp \left(-c N^{\frac{\alpha}{\log \log N}}\right)\right] .
\end{aligned}
$$

The conclusion of the proof immediately follows from (IV.14), (IV.15).

\section{Proof of Proposition 3}

We decompose the real line in the following way:

$$
\mathbb{R}=\Gamma_{1} \cup \Gamma_{2} \cup \Gamma_{3} \cup \Gamma_{4},
$$

where

$$
\begin{aligned}
& \left.\Gamma_{1}=\right]-\infty, \sqrt{N} \beta_{c}\left(1-\frac{1}{2 \beta_{c}^{2}} \frac{\log N}{N}+\frac{(1+\varepsilon)}{\beta_{c}^{2}} \frac{\log \log N}{N}\right], \\
& \left.\Gamma_{2}=\right] \sqrt{N} \beta_{c}\left(1-\frac{1}{2 \beta_{c}^{2}} \frac{\log N}{N}+\frac{(1+\varepsilon)}{\beta_{c}^{2}} \frac{\log \log N}{N}, \sqrt{N} \beta_{c}\right], \\
& \left.\left.\Gamma_{3}=\right] \sqrt{N} \beta_{c},\left(\left(\frac{1}{2 \beta_{c}^{2}}+\delta\right) \frac{\log N}{N}+1\right) \sqrt{N} \beta_{c}\right], \\
& \left.\Gamma_{4}=\right] \sqrt{N} \beta_{c}\left(1+\left(\frac{1}{2 \beta_{c}^{2}}+\delta\right) \frac{\log N}{N},+\infty[.\right.
\end{aligned}
$$


We have

$$
Z_{N} \leqq \sum_{K=1}^{4} \sum_{i=1}^{2^{N}} \mathbb{1}_{\Gamma_{K}}\left(X_{i}\right) \exp \beta \sqrt{N} X_{i}
$$

It follows from Lemma 7 below that:

$$
\operatorname{Prob}\left(\sum_{i=1}^{2^{N}} \mathbb{1}_{\Gamma_{1}}\left(X_{i}\right) \exp \beta \sqrt{N} X_{i}>\exp (N F(\beta))\right) \leqq \text { const } \exp -\frac{1}{\beta_{c}}(\log N)^{1+\varepsilon} \text {. }
$$

If the following inequalities are true:

$$
\begin{gathered}
\operatorname{Prob}\left(\sum_{i=1}^{2^{N}} \mathbb{1}_{\Gamma_{2}}\left(X_{i}\right) \geqq \exp 2 \log \log N\right) \leqq \text { const } \exp -(\log N)^{2}, \\
\operatorname{Prob}\left(\sum_{i=1}^{2^{N}} \mathbb{1}_{\Gamma_{3}}\left(X_{i}\right) \geqq \exp 2 \log \log N\right) \leqq \text { const } \exp -(\log N)^{2}, \\
\operatorname{Prob}\left(\sum_{i=1}^{2^{N}} \mathbb{1}_{\Gamma_{4}}\left(X_{i}\right)=0\right) \geqq 1-\frac{\text { const }}{N^{1+\delta}},
\end{gathered}
$$

then from Eqs. (IV.17)-(IV.20) we can deduce:

$$
\operatorname{Prob}\left(F_{N}(\beta) \geqq F(\beta)+\left(\frac{1}{2 \beta_{c}^{2}}+\delta\right) \frac{\log N}{N}+o\left(\frac{\log N}{N}\right)\right) \leqq \frac{\text { const }}{N^{1+\delta}},
$$

and so the first part of Proposition 3 is proven.

To prove inequalities (IV.18)-(IV.20) we first compute $\mathbb{E}\left(\mathbb{1}_{\Gamma_{K}}\right)$; we get

$$
\begin{aligned}
& \mathbb{E}\left(\mathbb{1}_{\Gamma_{2}}\right)=\frac{1}{\sqrt{2 \pi}} \underset{\sqrt{N} \beta_{c}\left(1-\frac{1}{2 \beta_{c}^{2}} \frac{\log N}{N}+\frac{(1+\varepsilon)}{\beta_{c}^{2}} \frac{\log \log N}{N}\right)}{\ln \beta_{c}} \exp -\frac{x^{2}}{2} d x \\
& \leqq \frac{1}{\beta_{c}}\left(\frac{1}{(\log N)^{1+\varepsilon}}-\frac{1}{\sqrt{N}}\right) \exp -\frac{\beta_{c}^{2}}{2} N \\
& \mathbb{E}\left(\mathbb{1}_{\Gamma_{3}}\right)=\frac{1}{\sqrt{2 \pi}} \int_{\sqrt{N} \beta_{c}}^{\left.\sqrt{N} \beta_{c}\left(1+\frac{\log N}{N}\left(\frac{1}{2 \beta_{c}}\right)+\delta\right)\right)} \exp -\frac{x^{2}}{2} d x \\
& \leqq \text { const } \frac{\log N}{\sqrt{N}} \exp -N \frac{\beta_{c}^{2}}{2}, \\
& \mathbb{E}\left(\mathbb{1}_{\Gamma_{4}}\right)=\frac{1}{\sqrt{2 \pi}} \int_{\sqrt{N} \beta_{c}\left(1+\frac{\log N}{N}\left(\frac{1}{2 \beta_{c}^{2}}+\delta\right)\right)}^{+\infty} \exp -\frac{x^{2}}{2} d x \\
& \leqq \frac{\text { const }}{\sqrt{N}}\left(\exp -\frac{\beta_{c}^{2}}{2} N\right)\left(\exp -\beta_{c}^{2}\left(\frac{1}{2 \beta_{c}^{2}}+\delta\right) \log N \leqq \text { const } \frac{\exp }{N^{1+\delta \beta_{c}^{2}}}-N \frac{\beta_{c}^{2}}{2}\right.
\end{aligned}
$$

Equations (IV.18), (IV.19) follow from the following inequality [VII]:

$$
\operatorname{Prob}\left(S_{n}>\imath\right) \leqq e(P(n) n)^{\imath},
$$


where $S_{n}=\sum_{i=1}^{n} \sigma_{i}$ and $\left\{\sigma_{i}\right\}_{i=1 \ldots n}$ is a family of independent identically distributed random variables with values in $\{0,1\}$ and such that:

$$
\operatorname{Prob}\left(\sigma_{i}=+1\right)=P(n) \text {. }
$$

To get the result it is sufficient to take $\sigma_{i}=\mathbb{1}_{\Gamma_{2}}\left(X_{i}\right), \sigma_{i}=\mathbb{1}_{\Gamma_{3}}\left(X_{i}\right)$, respectively, and $n=2^{N}$. Equation (IV.20) is a consequence of Markov's inequality [V] [see Eq. (III.14)].

Now we want to prove the second part of Proposition 3, namely, the upper bounds (II.7) on the quenched free energy.

From the previous arguments it is evident that $\forall \delta>0, \exists \Omega_{N}(\delta)$ with

$$
\mathbb{P}\left(\Omega_{N}(\delta)\right) \geqq 1-\frac{\text { const }}{N^{1+\alpha}}
$$

(for some positive $\alpha$ ) such that:

$$
\begin{aligned}
& \forall \omega \in \Omega_{N}(\delta): \\
& \quad \frac{1}{N} \log Z_{N}(\omega) \leqq F(\beta)+\left(\frac{1}{2 \beta_{c}^{2}}+\delta\right) \frac{\log N}{N} .
\end{aligned}
$$

Now recalling the definitions in (IV.12)

$$
\begin{aligned}
& \Omega_{+}=\left\{\omega \in \Omega: \log Z_{N} \geqq 0\right\}, \\
& \Omega_{-}=\left\{\omega \in \Omega: \log Z_{N}<0\right\},
\end{aligned}
$$

we can write:

$$
\begin{aligned}
\mathbb{E}\left(v \frac{1}{N} \log Z_{N}\right) \leqq & \mathbb{E}\left(\frac{1}{N} \log Z_{N} \mathbb{1}_{\Omega_{+} \cap \Omega_{N}(\delta)}\right) \\
& +\mathbb{E}\left(\frac{1}{N} \log Z_{N} \mathbb{1}_{\Omega_{+} \Omega_{N}^{\mathrm{f}}(\delta)}\right)
\end{aligned}
$$

We define

$$
\bar{\Omega}_{N}=\left\{\omega \in \Omega: \frac{\operatorname{Max}_{1 \leqq i \leqq 2^{N}} X_{i}}{\sqrt{N}}>2 \beta_{c}\right\},
$$

and so since

$$
\begin{gathered}
\frac{1}{N} \log Z_{N} \leqq \beta \frac{\operatorname{Max}_{1 \leqq i \leqq 2^{N}} X_{i}}{\sqrt{N}}+\log 2, \\
\mathbb{E}\left(\frac{1}{N} \log Z_{N} \mathbb{1}_{\Omega_{+} \cap \Omega_{N}^{c}(\delta)}\right) \\
\leqq \mathbb{E}\left(2 \beta \beta_{c} \mathbb{1}_{\Omega_{N}^{i}(\delta) \cap \bar{\Omega}_{N}}\right)+\mathbb{E}\left(\left(\frac{\operatorname{Max}_{1 \leqq i \leqq 2^{N}} X_{i}}{\sqrt{N}}+\log 2\right) \mathbb{1}_{\bar{\Omega}_{N}^{c}}\right) .
\end{gathered}
$$

From Eqs. (IV.25)-(IV.27), (IV.29), and (III.33) we get the desired result. 


\section{Proof of Proposition 4}

We decompose the real line in the following way:

$$
\begin{gathered}
\mathbb{R}=\bigcup_{K=-1}^{K(N)+1} \Delta_{K}, \\
\Delta_{-1}=\left[\sqrt{N} \beta_{c},+\infty[,\right. \\
\Delta_{0}=\left[\sqrt { N } \beta _ { c } \left(1-\frac{1}{2 \beta_{c}^{2}} \frac{\log N}{N}+\gamma_{N} \frac{\log N}{N}, \sqrt{N} \beta_{c}[,\right.\right. \\
\Delta_{K}=\left[\hat{X}_{K+1}, \hat{X}_{K}[\text { if } K=1, \ldots, K(N)\right.
\end{gathered}
$$

with

$$
\hat{X}_{K}=\sqrt{N} \beta_{c}\left(1-\frac{1}{2 \beta_{c}^{2}} \frac{\log N}{N}-(K-1) \gamma_{N} \frac{\log N}{N}\right)
$$

and $K(N)=\left[\frac{N}{\gamma_{N} \log N}\right]$,

We have

$$
\Delta_{K(N)+1}=\mathbb{R} \backslash\left(\bigcup_{K=-1}^{K(N)} \Delta_{K}\right)
$$

$$
\begin{aligned}
Z_{N} \leqq & \sum_{K=1}^{K(N)}\left(\exp \sqrt{N} \beta \hat{X}_{K}\right) \sum_{i=1}^{2^{N}} \mathbb{1}_{\Delta_{K}}\left(X_{i}\right)+\sum_{i=1}^{2^{N}} \mathbb{1}_{\Delta_{K(N)+1}}\left(X_{i}\right) \\
& +\sum_{i=1}^{2^{N}} \mathbb{1}_{\Delta_{-1}}\left(X_{i}\right) \exp \sqrt{N} \beta X_{i}+\sum_{i=1}^{2^{N}} \mathbb{1}_{\Delta_{0}}\left(X_{i}\right) \exp \left(N \cdot \beta \beta_{c}\right)
\end{aligned}
$$

We first consider the third sum in the right-hand side of (IV.31):

$$
\begin{aligned}
\mathbb{P}( & \left.\sum_{i=1}^{2^{N}} \mathbb{1}_{\Delta_{-1}}\left(X_{i}\right) \exp \sqrt{N} \beta X_{i} \geqq \frac{1}{3} \exp N \beta \cdot \beta_{c}\left(1-\frac{1}{2 \beta_{c}^{2}} \frac{\log N}{N}\right)\right) \\
\leqq & \mathbb{P}\left(\left(\exp \sqrt{N} \beta \operatorname{Max}_{1 \leqq i \leqq 2^{N}} X_{i}\right)\left(\sum_{i=1}^{2^{N}} \mathbb{1}_{\Delta_{-1}}\left(X_{i}\right)\right) \geqq \frac{1}{3} \exp N \beta \cdot \beta_{c}\left(1-\frac{1}{2 \beta_{c}^{2}} \frac{\log N}{N}\right)\right) \\
= & \mathbb{P}\left(( \operatorname { e x p } \sqrt { N } \beta \operatorname { M a x } _ { 1 \leqq i \leqq 2 ^ { N } } X _ { i } ) \left(\sum_{i=1}^{2^{N}} \mathbb{1}_{\Delta_{-1}}\left(X_{i}\right) \geqq \frac{1}{3} \exp N \beta \cdot \beta_{c}\left(1-\frac{1}{2 \beta_{c}^{2}} \frac{\log N}{N}\right)\right.\right. \\
& \left.\sum_{i=1}^{2^{N}} \mathbb{1}_{\Delta_{-1}}\left(X_{i}\right) \geqq 1\right) \\
\leqq & \mathbb{P}\left(\sum_{i=1}^{2^{N}} \mathbb{1}_{\Delta_{-1}}\left(X_{i}\right) \geqq 1\right)=1-\left[1-\mathbb{E}\left(\mathbb{1}_{\Delta_{-1}}\right)\right]^{2^{N}} .
\end{aligned}
$$

It is easy to see that the right-hand side of (IV.32) does not exceed $\frac{\text { const }}{\sqrt{N}}$, therefore,

$$
\mathbb{P}\left(\sum_{i=1}^{2^{N}} \mathbb{1}_{\Delta_{-1}}\left(X_{i}\right) \exp \sqrt{N} \beta X_{i} \geqq \frac{1}{3} \exp N \beta \beta_{c}\left(1-\frac{1}{2 \beta_{c}^{2}} \frac{\log N}{N}\right)\right) \leqq \frac{\text { const }}{\sqrt{N}}
$$


Now we consider the last sum in the right-hand side of (IV.31):

$$
\begin{aligned}
& \mathbb{P}\left(\left(\exp N \beta \beta_{c}\right) \sum_{i=1}^{2^{N}} \mathbb{1}_{\Delta_{0}}\left(X_{i}\right) \geqq \frac{1}{3} \exp N \beta \beta_{c}\left(1-\frac{1}{2 \beta_{c}^{2}} \frac{\log N}{N}\right)\right) \\
& \quad=\mathbb{P}\left(\sum_{i=1}^{2^{N}} \mathbb{1}_{\Delta_{0}}\left(X_{i}\right) \geqq \frac{1}{3} \exp -\frac{\beta}{2 \beta_{c}^{2}} \log N ; \sum_{i=1}^{2^{N}} \mathbb{1}_{\Delta_{0}}\left(X_{i}\right) \geqq 1\right) \\
& \quad \leqq \operatorname{Prob}\left(\sum_{i=1}^{2^{N}} \mathbb{1}_{\Delta_{0}}\left(X_{i}\right) \geqq 1\right)=1-\left(1-\mathbb{E}\left(\mathbb{1}_{\Delta_{0}}\right)\right)^{2^{N}} \\
& \quad \leqq 1-\exp -2^{N-1} \mathbb{E}\left(\mathbb{1}_{\Delta_{0}}\right) .
\end{aligned}
$$

A simple change of variable implies that

$$
2^{N} \mathbb{E}\left(\mathbb{1}_{\Delta_{0}}\right) \leqq \frac{1}{\beta_{c}}\left[\left(\exp -\gamma_{N} \beta_{c}^{2} \log N\right)-\frac{1}{\sqrt{N}}\right] .
$$

Therefore, if we choose $\gamma_{N}=\frac{(1+\varepsilon)}{\beta_{c}^{2}} \frac{\log \log N}{\log N}$, we get

$$
\begin{aligned}
& \mathbb{P}\left(\left(\exp N \beta \cdot \beta_{c}\right) \sum_{i=1}^{2 N} \mathbb{1}_{\Delta_{0}}\left(X_{i}\right) \geqq \frac{1}{3} \exp N \beta \beta_{c}\left(1-\frac{1}{2 \beta_{c}^{2}} \frac{\log N}{N}\right)\right) \\
& \quad \leqq \frac{\text { const }}{\beta_{c}}\left(\frac{1}{(\log N)^{1+\varepsilon}}-\frac{1}{\sqrt{N}}\right) .
\end{aligned}
$$

In the following lemma we study the first sums in the right-hand side of (IV.31).

\section{Lemma 7.}

$$
\begin{aligned}
& \mathbb{P}\left(\sum_{K=1}^{K(N)}\left(\exp \sqrt{N} \beta \hat{X}_{K}\right) \sum_{i=1}^{2^{N}} \mathbb{1}_{\Delta_{K}}\left(X_{i}\right)+\sum_{i=1}^{2 N} \mathbb{1}_{\Delta_{K(N)+2}}\left(X_{i}\right)\right. \\
& \left.\geqq \beta_{c} \gamma_{N} \log N \exp N \beta \beta_{c}\left(1-\frac{1}{2 \beta_{c}^{2}} \frac{\log N}{N}\right)\right) \\
& \quad \leqq \text { const } \exp -\frac{1}{\beta_{c}}(\log N)^{1+\varepsilon} .
\end{aligned}
$$

Proof of Lemma 7. If we use the Markov inequality [V] and the fact that $\mathbb{E}\left(\Delta_{K(N)+1}\right) \leqq \frac{1}{2}$ it is straightforward that:

$$
\begin{aligned}
& \mathbb{P}\left(\sum_{i=1}^{2^{N}} \mathbb{1}_{\Delta_{K(N)+1}}\left(X_{i}\right) \geqq \exp N\left(\beta \cdot \beta_{c}\right)\left(1-\frac{1}{2 \beta_{c}^{2}} \frac{\log N}{N}\right)\right) \\
& \quad \leqq \exp \left(\frac{\beta}{2 \beta_{c}} \log N-N\left(\beta-\frac{\beta_{c}}{2}\right) \beta_{c}\right) .
\end{aligned}
$$

On the other hand, it is a direct consequence of the Bernstein inequality that for any $1 \leqq K \leqq K(N)$,

$$
\begin{aligned}
& \mathbb{P}\left(\sum_{i=1}^{2^{N}} \mathbb{1}_{\Delta_{K}}\left(X_{i}\right) \geqq 2^{N+1} \mathbb{E}\left(\mathbb{1}_{\Delta_{K}}\right)\right) \\
& \quad \leqq \mathbb{P}\left(\left|\sum_{i=1}^{2^{N}}\left(\mathbb{1}_{\Delta_{K}}\left(X_{i}\right)-\mathbb{E}\left(\mathbb{1}_{\Delta_{K}}\left(X_{i}\right)\right)\right)\right| \geqq 2^{N} \mathbb{E}\left(\mathbb{1}_{\Delta_{K}}\right)\right) \\
& \quad \leqq \exp -2^{N-1} \mathbb{E}\left(\mathbb{1}_{\Delta_{K}}\right) .
\end{aligned}
$$


Therefore,

$$
\begin{aligned}
& \mathbb{P}\left(\sum_{K=1}^{K(N)}\left(\exp \sqrt{N} \beta \hat{X}_{K}\right) \sum_{i=1}^{2^{N}} \mathbb{1}_{\Delta_{K}}\left(X_{i}\right) \geqq \sum_{K=1}^{K(N)} 2^{N+1} \mathbb{E}\left(\mathbb{1}_{\Delta_{K}}\right) \exp \sqrt{N} \beta \hat{X}_{K}\right) \\
& \quad \leqq \sum_{K=1}^{K(N)} \exp -2^{N-1} \mathbb{E}\left(\mathbb{1}_{\Delta_{K}}\right) .
\end{aligned}
$$

Using

$$
\mathbb{E}\left(\mathbb{1}_{\Delta_{K}}\right) \leqq \beta_{c} \gamma_{N} \frac{\log N}{\sqrt{N}} \exp -N \frac{\beta_{c}^{2}}{2}\left(1-\frac{1}{2 \beta_{c}^{2}} \frac{\log N}{N}-\gamma_{N}(K-1) \frac{\log N}{N}\right)^{2},
$$

we get

$$
\begin{aligned}
& \sum_{K=1}^{K(N)} 2^{N} \mathbb{E}\left(\mathbb{1}_{\Delta_{\mathrm{K}}}\right) \exp \sqrt{N} \beta \hat{X}_{K} \\
& \leqq C_{1} \beta_{c} \gamma_{N} \log N \exp \left\{\beta \beta_{c} N\left(1-\left(\frac{1}{2 \beta_{c}^{2}}-\gamma_{N}\right) \frac{\log N}{N}\right)-\frac{1}{2}\left(\frac{\log N}{N}\right) N\right\} \\
& \cdot \exp N\left(\beta_{c}^{2}\left(\frac{1}{2 \beta_{c}^{2}}-\gamma_{N}\right) \frac{\log N}{N}\right. \\
& \cdot \sum_{K=1}^{K(N)} \exp -K N\left\{\beta \beta_{c} \gamma_{N} \frac{\log N}{N}-\beta_{c}^{2} \gamma_{N} \frac{\log N}{N}+\gamma_{N}^{2} \frac{\beta_{c}^{2}}{2} \frac{(K-1)^{2}}{K}\left(\frac{\log N}{N}\right)^{2}\right\} .
\end{aligned}
$$

The last sum in the right-hand side of (IV.40) does not exceed

$$
\text { const } \exp -\gamma_{N}\left(\beta \beta_{c}-\beta_{c}^{2}\right) \log N \text {. }
$$

Therefore,

$$
\sum_{K=1}^{K(N)} 2^{N} \mathbb{E}\left(\mathbb{1}_{\Delta_{K}}\right) \exp \sqrt{N} \beta \hat{X}_{K} \leqq \text { const } \gamma_{N} \log N \exp N \beta \beta_{c}\left(1-\frac{1}{2 \beta_{c}^{2}} \frac{\log N}{N}\right) .
$$

On the other hand, if we use

$$
\mathbb{E}\left(\mathbb{1}_{\Delta_{K}}\right) \geqq \frac{1}{\beta_{c}} 2^{-N}\left(\exp \beta_{c}^{2} K \gamma_{N} \log N\right)\left(1-\exp -\beta_{c}^{2} \gamma_{N} \log N\right) \exp -o\left(\left(\frac{\log N}{N}\right)^{2}\right),
$$

we get

$$
\sum_{K=1}^{K(N)} \exp -2^{N} \mathbb{E}\left(\mathbb{1}_{\Delta_{K}}\right) \leqq \text { const } \exp -\frac{1}{2 \beta_{c}} \exp \beta_{c}^{2} \gamma_{N} \log N
$$

Using $\gamma_{N}=\frac{(1+\varepsilon)}{\beta_{c}^{2}} \frac{\log \log N}{\log N},($ IV.37), (IV.39), (IV.41)-(IV.43), we get the result.

Now the Proposition 4 is a direct consequence of (IV.33), (IV.35), and Lemma 7. 
4. Proof of Proposition 5

Define: $\bar{X}=\frac{\beta}{\beta_{c}}<1, \theta=\frac{1-\bar{X}}{2}$,

$$
\begin{aligned}
& K_{-}=\left[(\bar{X}-\theta) \frac{\sqrt{N} \beta_{c}}{\gamma_{N}}\right], \\
& K_{+}=\left[(\bar{X}+\theta) \frac{\sqrt{N} \beta_{c}}{\gamma_{N}}\right]-1,
\end{aligned}
$$

where $\gamma_{N} \underset{N \rightarrow \infty}{\longrightarrow} 0$, and will be specified later.

$$
\Delta_{K}=\left[K \gamma_{N},(K+1) \gamma_{N}[\right.
$$

for $K=K_{-}, K_{-}+1, \ldots, K_{+}$,

$$
\bar{\Delta}=\mathbb{R} \backslash\left(\bigcup_{K=K_{-}}^{K_{+}} \Delta_{K}\right)
$$

We have

$$
\begin{aligned}
\sum_{K=K_{-}}^{K_{+}} \sum_{i=1}^{2^{N}} \mathbb{1}_{\Delta_{K}}\left(X_{i}\right) \exp \sqrt{N} \beta X_{i} \leqq & Z_{N} \leqq \sum_{K=K_{-}}^{K_{+}} \sum_{i=1}^{2^{N}} \mathbb{1}_{\Delta_{K}}\left(X_{i}\right) \exp \sqrt{N} \beta X_{i} \\
& +\sum_{i=1}^{2^{N}} \mathbb{1}_{\bar{\Delta}}\left(X_{i}\right) \exp \sqrt{N} \beta X_{i}
\end{aligned}
$$

Let

$$
\begin{aligned}
& \Omega_{N}^{(1)}=\left\{\left|\sum_{i=1}^{2^{N}}\left(\mathbb{1}_{\Delta_{K}}\left(X_{i}\right)-\mathbb{E}\left(\mathbb{1}_{\Delta_{K}}\left(X_{i}\right)\right)\right)\right|>\varepsilon_{N} 2^{N} \mathbb{E}\left(\mathbb{1}_{\Delta_{K}}\right), \forall K=K_{-}, \ldots, K_{+}\right\}, \\
& \Omega_{N}^{(2)}=\left\{\sum_{i=1}^{2^{N}} \mathbb{1}_{\bar{\Delta}}\left(X_{i}\right) \exp \sqrt{N} \beta X_{i}>\exp N G_{\beta}(\bar{x}+\theta)+q_{\beta}\right\},
\end{aligned}
$$

where $0<\varepsilon_{N} \underset{N \rightarrow \infty}{\longrightarrow} 0$ and will be chosen later;

$$
q_{\beta}=\frac{1}{2}\left(F(\beta)-G_{\beta}(\bar{x}+\theta)\right)>0 .
$$

For any $\omega \in \Omega_{N}^{(1)} \cap \Omega_{N}^{(2)}$, we have

$$
\begin{aligned}
& \sum_{K=K_{-}}^{K_{+}}\left(1-\varepsilon_{N}\right) 2^{N}\left(\exp k \beta \gamma_{N} \sqrt{N}\right) \mathbb{E}\left(\mathbb{1}_{\Delta_{K}}\right) \\
& \leqq Z_{N} \leqq \sum_{K=K_{-}}^{K_{+}}\left(1+\varepsilon_{N}\right) 2^{N}\left(\exp (K+1) \beta \gamma_{N} \sqrt{N}\right) \mathbb{E}\left(\mathbb{1}_{\Delta_{K}}\right) \\
& \quad+\exp N\left[G_{\beta}(\bar{x}+\theta)+q_{\beta}\right] .
\end{aligned}
$$

Now since

$$
\gamma_{N} \frac{1}{\sqrt{2 \pi}} \exp -\frac{(K+1)^{2} \gamma_{N}^{2}}{2} \leqq \mathbb{E}\left(\mathbb{1}_{\Delta_{K}}\right) \leqq \frac{\gamma_{N}}{\sqrt{2 \pi}} \exp -\frac{K^{2} \gamma_{N}^{2}}{2}
$$

then for $\omega \in \Omega_{N}^{(1)} \cap \Omega_{N}^{(2)}$, 


$$
\begin{aligned}
& {\left[\sum_{K=K_{-}}^{K_{+}} G_{-}(K) \gamma_{N}\right] 2^{N} \exp -\gamma_{N} \beta \sqrt{N}-2 K_{+} \gamma_{N}^{2}-\gamma_{N}^{2}} \\
& \quad \leqq Z_{N} \leqq\left[\sum_{K=K_{-}}^{K_{+}} G_{+}(K) \gamma_{N}\right] 2^{N} \exp \gamma_{N} \beta \sqrt{N}+2 K_{+} \gamma_{N}^{2}+\gamma_{N}^{2},
\end{aligned}
$$

where $G_{-}(K)=\frac{1}{\sqrt{2 \pi}} \exp \beta \sqrt{N} \gamma_{N}(K+1)-(K+1)^{2} \frac{\gamma_{N}^{2}}{2}$,

$$
G_{+}(K)=\frac{1}{\sqrt{2 \pi}} \exp \beta \sqrt{N} \gamma_{N} K-\frac{(K)^{2} \gamma_{N}^{2}}{2}
$$

and, for $x \in \Delta_{K}$

$$
G_{+}(K) \leqq \frac{1}{\sqrt{2 \pi}} \exp \beta \sqrt{N} x-\frac{x^{2}}{2} \leqq G_{-}(K)
$$

so that, recalling that $\frac{\beta_{c}^{2}}{2}=\log 2$, we get

$$
\begin{aligned}
& {\left[\int_{(\bar{x}-\theta) \sqrt{N} \beta_{c}}^{(\bar{x}+\theta) \sqrt{N} \beta_{c}}\left(\exp \beta \sqrt{N} x-\frac{x^{2}}{2}\right) \frac{d x}{\sqrt{2 \pi}}\right]\left(\exp \frac{\beta_{c}^{2}}{2} N\right)} \\
& \cdot\left(\exp -\gamma_{N} \sqrt{N}\left(\beta+2(\bar{x}+\theta)+\frac{\gamma_{N}}{\sqrt{N}}\right)\right) \\
& \leqq Z_{N} \leqq\left[\int_{-\infty}^{+\infty}\left(\exp \beta \sqrt{N} x-\frac{x^{2}}{2}\right) \frac{d x}{\sqrt{2 \pi}}\right] \\
& \cdot\left(\exp \frac{\beta_{c}^{2}}{2} N\right)\left(\exp \gamma_{N} \sqrt{N}\left(\beta+2(\bar{x}+\theta)+\frac{\gamma_{N}}{\sqrt{N}}\right)\right) \\
& +\exp N\left(G_{\beta}(\bar{x}+\theta)+q_{\beta}\right) .
\end{aligned}
$$

From which by an easy calculation we get:

$$
\begin{aligned}
& \left\{\left[\exp -\gamma_{N} \sqrt{N}\left(\beta+2(\bar{x}+\theta)+\frac{\gamma_{N}}{\sqrt{N}}\right)\right][\exp +N F(\beta)]\right\}\left(1-2 \exp -\theta \frac{\beta_{c}^{2}}{2} N\right) \\
& \quad \leqq Z_{N} \leqq\left\{\left[\exp \gamma_{N} \sqrt{N}\left(\beta+2(\bar{x}+\theta)+\frac{\gamma_{N}}{\sqrt{N}}\right)\right][\exp N F(\beta)]\right\}\left(1+e^{-q_{\beta} N}\right)
\end{aligned}
$$

Now we claim that $\forall \beta<\beta_{c}, \exists \xi>0$ :

$$
\mathbb{P}\left(\Omega_{N}^{(1)}\right) \geqq 1-\frac{2 \theta \sqrt{N} \beta_{c}}{\gamma_{N}} \exp -\frac{1}{8} \varepsilon_{N}^{2} \exp \xi N .
$$

In order to obtain Eq. (IV.53) it is sufficient to show that $\forall K=K_{-}, \ldots, K_{+}$,

$$
\begin{aligned}
& \mathbb{P}\left(\left|\sum_{i=1}^{2^{N}} \mathbb{1}_{\Delta_{K}}\left(X_{i}\right)-\mathbb{E}\left(\mathbb{1}_{\Delta_{K}}\left(X_{i}\right)\right)\right|>\varepsilon_{N} 2^{N} \mathbb{E}\left(\mathbb{1}_{\Delta_{K}}\right)\right) \\
& \quad \leqq \exp -\frac{\varepsilon_{N}^{2}}{8} 2^{N} \mathbb{E}\left(\mathbb{1}_{\Delta_{K}}\right),
\end{aligned}
$$


since it follows from the definition of $K_{-}, K_{+}$that:

$$
\inf _{K-\leqq K \leqq K_{+}} \mathbb{E}\left(\mathbb{1}_{\Delta_{K}}\right) \geqq 2^{-N} \exp \xi N
$$

for some positive constant $\xi$.

Equation (IV.54) is again a consequence of Bernstein's inequality (see the proof of Lemma 6 above).

It is easy to see by an argument very similar to the one used in the proof of Theorem 1 that $\exists \mu>0$, such that

$$
\mathbb{P}\left(\Omega_{N}^{(2)}\right)>1-\exp -\mu N .
$$

In fact, the quantity $\sum_{i=1}^{2^{N}} \mathbb{1}_{\bar{\Delta}}\left(X_{i}\right) \exp \beta \sqrt{N} X_{i}$ appearing in the definition of $\Omega_{N}^{(2)}$ is a sort of partition function evaluated in the "restricted ensemble" that excludes the energy levels in the interval $[\bar{x}-\theta, \bar{x}+\theta] \sqrt{N} \beta_{c}$; so we can apply the analog of the heuristic argument given in the introduction (namely, the maximization of $G_{\beta}(x)$ in $[0,1] \backslash[\bar{x}-\theta, \bar{x}+\theta]$ as well as the rigorous estimates (III.4), (III.7), (III.12), (III.14), to get (IV.55).

Now if we choose $\varepsilon_{N}=\exp -\xi \frac{N}{4}$ and for example, $\gamma_{N}=\exp -N$, then by Eqs. (IV.52), (IV.53), (IV.55), using again Borel-Cantelli's lemma, we conclude the proof of the first part of Proposition 5.

To get the other result concerning the quenched free energy, namely Eq. (II.10), we proceed exactly like in the case $\beta \geqq \beta_{c}$ but now, we use the exponentially decreasing estimates (IV.53), (IV.55), instead of the power like bounds of the case $\beta \geqq \beta_{c}$.

Finally, if we apply the explicit bound (III.33) to evaluate

$$
\mathbb{E}\left[\left(\operatorname{Max}_{1 \leqq i \leqq 2^{N}} \frac{X_{i}}{\sqrt{N}}+\log 2\right) \mathbb{1}_{\bar{\Omega}_{N}}\right]
$$

[see Eq. (IV.20)] we get the result.

Remark 1. It follows from Eqs. (III.4) and (III.14) that $F_{N}(\beta)$ is geometrically bounded from above by $F(\beta)$ for all $\beta>0$ (see Definition 4.1 of [IV]). On the other hand, we get from (III.24) that $F_{N}(\beta)$ is geometrically bounded from below by $F(\beta)$ for all $\beta>0$. These two facts imply geometric convergence for all $\beta>0$ (Definition 4.1 of [IV]).

This convergence was proved in [IV] for $\beta \leqq \beta_{c^{*}}$. In the same paper, it was conjectured that the geometric convergence fails for $\beta>\beta_{c}$. Our method allows us to disprove this conjecture.

Remark 2. It is not difficult to see by using Bernstein's inequality that $F_{N}(\beta)$ is hypergeometrically bounded from below by $F(\beta)$ (Definition 4.2 of [IV]) for any $\beta>0$.

We can also prove the following statement: For all $\beta>0, F_{N}(\beta)$ is not hypergeometrically bounded from above by $F(\beta)$. The reason is the following:

$$
\mathbb{P}\left(F_{N}(\beta) \geqq 2 F(\beta)\right) \geqq \mathbb{P}\left(Z_{N} \geqq \exp N \beta \beta_{c} y\right) \geqq \mathbb{P}\left(\sum_{i=1}^{2^{N}} \mathbb{1}_{\left[\beta_{c} y \sqrt{N},+\infty[\right.}\left(X_{i}\right) \geqq 1\right),
$$


if $y$ is such that $\beta \beta_{c} y>2 F(\beta)$, but

$$
\begin{aligned}
\mathbb{P}\left(\sum_{i=1}^{2^{N}} \mathbb{1}_{\left[\beta_{c} y \sqrt{N},+\infty[\right.}\left(X_{i}\right) \geqq 1\right) & =1-\left(1-\operatorname{Prob}\left(X \geqq \beta_{c} y \sqrt{N}\right)\right)^{2^{N}} \\
& \geqq \operatorname{const} 2^{N} \exp -\beta_{c}^{2} y^{2} \frac{N}{2}
\end{aligned}
$$

and so $F_{N}(\beta)$ can be at most geometrically but not hypergeometrically bounded from above by $F(\beta)$.

Remark 3. By straightforward calculations it is easy to extend the results contained in Theorem 1 to the case of models describing by the partition function

$$
Z_{N}=\sum_{i=1}^{\kappa^{N}} \exp (N)^{1 / p} \beta X_{i},
$$

where $X_{i}$ are independent identically distributed random variables with

$$
\operatorname{Prob}\left(X_{i} \geqq x\right) \approx \exp -b x^{\frac{p}{p-1}}
$$

for $x \rightarrow \infty$ for some positive $b$ and $p \in[1,+\infty[$ (see [IV]).

Acknowledgements. One of us (E.O.) wishes to express thanks for the very kind hospitality of the "Centre de Physique Théorique Luminy" and the "Université de Provence".

\section{References}

I Derrida, B.: Random energy model: Limit of a family of disordered models. Phys. Rev. Lett. 45, 79-82 (1980)

II Derrida, B. Random energy model: An exactly solvable model of disordered systems. Phys. Rev. B 24, 2613-2626 (1981)

III Sherrington, D., Kirkpatrick, S.: Solvable model of a spin-glass. Phys. Rev. Lett. 35, 1792-1796 (1975)

IV Eisele, Th.: On a third order phase transition. Commun. Math. Phys. 90, 125-159 (1983)

V Chow, Y.S., Teicher, H.: Probability theory. Berlin, Heidelberg, New York: Springer 1978

VI Petrov, V.V.: Sums of independent random variables. Berlin, Heidelberg, New York: Springer 1975, p. 55

VII Orey, S., Taylor, J.: Proc. London Math. Soc. 3, 174-192 (1974), Lemma 2.1

Communicated by T. Spencer

Received April 19, 1984 